IdeAs

Idées d'Amériques

$14 \mid 2019$

Populismes dans les Amériques

\title{
Du populisme " par le haut » au populisme « par le bas ». Les apports d'une enquête de terrain à la redéfinition d'un concept flou
}

From populism "from above" to populism "from below". An empirical contribution to a new analysis of an all-embracing a vague concept Del populismo «por arriba» al populismo «por debajo». Los aportes de un trabajo de campo a la redefinición de un concepto frágil

\section{Federico Tarragoni}

\section{OpenEdition}

Journals

Édition électronique

URL : http://journals.openedition.org/ideas/6780

DOI : $10.4000 /$ ideas. 6780

ISSN : $1950-5701$

Éditeur

Institut des Amériques

Référence électronique

Federico Tarragoni, «Du populisme «par le haut » au populisme «par le bas ». Les apports d'une enquête de terrain à la redéfinition d'un concept flou », IdeAs [En ligne], 14 | 2019, mis en ligne le 01 octobre 2019, consulté le 08 novembre 2019. URL : http://journals.openedition.org/ideas/6780 ; DOI : $10.4000 /$ ideas. 6780

Ce document a été généré automatiquement le 8 novembre 2019

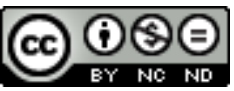

IdeAs - Idées d'Amériques est mis à disposition selon les termes de la licence Creative Commons Attribution - Pas d'Utilisation Commerciale - Pas de Modification 4.0 International. 


\title{
Du populisme « par le haut » au populisme «par le bas ». Les apports d'une enquête de terrain à la redéfinition d'un concept flou
}

\author{
From populism "from above" to populism "from below". An empirical \\ contribution to a new analysis of an all-embracing a vague concept \\ Del populismo «por arriba» al populismo «por debajo». Los aportes de un trabajo \\ de campo a la redefinición de un concepto frágil
}

Federico Tarragoni

\section{Du populisme « par le haut » au populisme « par le bas ». Les apports d'une enquête de terrain à la redéfinition d'un concept flou}

\footnotetext{
La science politique le reconnaît depuis un demi-siècle : le concept de populisme est problématique (Gellner E. et Ionescu G., 1969 ; Canovan M., 1981 ; Dézé A., 2004). Utilisé comme une insulte, souvent confondu avec la démagogie, il est aujourd'hui employé comme un terme fourre-tout permettant de désigner des mouvements d'extrême gauche et d'extrême droite, aux histoires et aux idéologies difficilement comparables. Les sciences sociales sont désormais face à un choix cornélien : se débarrasser de ce concept plus normatif que positif (Tarragoni F., 2013a), ou le redéfinir drastiquement afin d'en restreindre l'usage à quelques cas sociologiquement pertinents. Parmi eux, l'Amérique latine occupe une place absolument centrale. Si quelque part on peut trouver une définition idéale-typique du populisme, c'est dans l'histoire latinoaméricaine qu'il faut la chercher. Cela pour la simple et bonne raison que ce n'est qu'en Amérique latine que le populisme est parvenu au pouvoir et que, entre le $\mathrm{XX}^{\mathrm{e}}$ et le $\mathrm{XXI}^{\mathrm{e}}$ siècle, il a donné lieu à une tradition politique singulière. Le populisme latino-
} 
américain n'est pas une variante exceptionnelle du phénomène, comme on l'a longtemps cru en en faisant le "merle blanc» de la tradition populiste mondiale (QuattrocchiWoisson D., 1997, 162-163). Il est, avec le narodnischestvo russe (1840-80) et le People's Party étatsunien (1877-96), le seul populisme qu'on puisse vraiment désigner comme tel.

Une telle proposition pourrait paraître incongrue à certains, vu que, depuis trente ans, nous avons contracté la fâcheuse habitude de voir dans les partis ultranationalistes et xénophobes à l'échelle du monde, les étendards du nouveau populisme planétaire. Qu'est-ce que ce défilé inépuisable de démagogues aux tendances autoritaires, chassant partout les minorités - ethniques, culturelles, sexuelles -, a toutefois à voir avec les narodniki, le People's Party ou la tradition populiste latino-américaine? En réalité pas grand-chose, si ce n'est la permanence historique de l'opposition discursive "peuple vs élite ", censée avoir été « relue » dans les termes «nation vs étranger » (Hermet G., 2001 ; Taguieff P.-A., 2007).

Une telle permanence, à elle seule, ne saurait légitimer la comparaison. Une partie de la science politique contemporaine a de facto confondu, et continue toujours de confondre nationalisme et populisme (Villacañas Berlanga J., 2017, 21-24). L'extrême diversité des projets politiques, anciens et actuels, réunis sous l'étiquette de "populisme", est si frappante que certains politistes n'hésitent pas aujourd'hui à invalider la comparaison. Mais ils le font, pour ainsi dire, «à rebours ». Au lieu de penser le populisme à partir de ses manifestations "sûres" - celles du passé sur lesquelles nous avons une ample documentation historique -, ils font exactement l'inverse : ils soustraient le populisme à toute historicité. Prenons Jan-Werner Müller, auteur de Qu'est-ce que le populisme? Définir enfin la menace : « un certain nombre de mouvements du passé, écrit-il, qui sont toujours présentés comme "populistes" ne l'étaient pas, pour l'essentiel, selon l'acception que je propose ici. [...] Il faut donc tout d'abord nettoyer le concept de populisme des significations qu'il a fréquemment revêtues dans l'histoire sociale. [...] Une fois ce concept nettoyé des significations qu'il a pu revêtir dans le passé, il apparaît que le populisme représente un danger spécifique, inhérent à la démocratie représentative moderne" (Müller J.-W., 2018, 32-34). Une telle méthode semble toutefois aux antipodes des sciences sociales: comment analyser un phénomène politique sans prendre en compte son historicité ? Ce «nettoyage» des «significations de l'histoire sociale» ne relève-t-il pas, tout simplement, de l'amnésie et du présentisme?

4 C'est pourquoi, lorsqu'on cherche à comprendre ce qu'est le populisme, le recours à l'Amérique latine est fondamental. Si celle-ci fut, avec la Russie et les États-Unis, une terre d'élection du populisme - le "vrai », pas celui «en pacotille» de nos leaders xénophobes - c'est que la différence avec le nationalisme identitaire y fut très claire. Les oppositions "peuple vs élite» et "nation vs étranger» ne furent guère interchangeables. Entre les années 1930 et 1960, les populismes « classiques »- dont le péronisme argentin est la matrice -, donnent de la nation une définition plébéienne et anti-impérialiste. Comme le répétaient à l'envie les militants péronistes, le combat de l'« Argentin » était assimilé à celui de toutes les nations opprimées par l'« Empire » - les puissances coloniales puis néocoloniales ${ }^{1}$. La marque du populisme en Amérique latine n'a jamais été l'ultranationalisme xénophobe que ses théoriciens contemporains lui reprochent. C'était, et c'est encore un projet de démocratisation de républiques libérales caractérisées par de forts dualismes sociaux (Touraine A., 1988). Voici le projet politique commun à l'yrigoyenismo (du nom du président Yrigoyen) puis au péronisme 
argentin, au cardénisme mexicain (du nom du président Cárdenas), au gétulisme brésilien (du nom du président Getúlio Vargas), au movimientismo bolivien (du nom du parti « Movimiento Nacional Revolucionario »), au velasquisme équatorien (du nom du président Velasco Ibarra) et péruvien (du nom du président Velasco Alvarado), à l'adécisme vénézuélien (du nom du parti Acción democrática), au gaitanisme colombien (du nom du leader assassiné Gaitán), à l'ibañismo chilien (du nom du président Ibáñez del Campo), à l'aprisme péruvien (du nom du parti APRA).

Ces différentes expériences politiques sont qualifiées dans l'histoire latino-américaine comme autant d'" ismes ", car elles ne résument pas à des partis ou à des personnalités politiques marquantes. Elles singularisent l'histoire politique de leurs pays respectifs et du sous-continent en général : les gouvernements auxquels la majorité d'entre elles ont donné lieu, ont tous cherché à démocratiser des républiques libérales - oligarchiques, inégalitaires et corrompues. Comment? En élargissant le suffrage démocratique, tout d'abord : il suffit de penser à l'élection au suffrage universel de Vargas, Perón, Velasco Ibarra et Ibáñez del Campo; en créant, ensuite, les conditions économiques d'une véritable autonomie par rapport aux ingérences néocoloniales, grâce à la nationalisation des ressources stratégiques et/ou à l'industrialisation substitutive des importations; en mettant en place, enfin, un système de sécurité et de protection sociale, fondé sur des droits collectifs pour les travailleurs.

6 Ce populisme classique est mort avec les dictatures militaires des années 1970, puis il a été définitivement enterré, en tant que projet politique, par le néolibéralisme des années 1990. Mais il a connu un «revival » au début des années 2000, dans une partie dudit «tournant à gauche » latino-américain : les gouvernements d'Hugo Chávez au Venezuela, d'Evo Morales en Bolivie et de Rafael Correa en Équateur. De ces trois expériences, celle du Venezuela est sans doute la plus emblématique. Elle a assumé, au $\mathrm{XXI}^{\mathrm{e}}$ siècle, le même rôle de modèle que le péronisme au $\mathrm{XX}^{\mathrm{e}}$. Elle a incarné la réinvention idéologique et programmatique du populisme latino-américain. Or, tout comme le projet démocratique péroniste fut fragilisé, avant d'être liquidé manu militari par la Junta, par l'autoritarisme croissant de Perón lui-même, le projet démocratique chaviste a été affaibli par les "néo-bolivariens" au pouvoir. Après le passage au " socialisme du XXI ${ }^{e}$ siècle » (2005) et, surtout, lors de la transition de Chávez à Maduro (2013), le régime bolivarien a entamé un virage décidément autoritaire. La polarisation interne entre "peuple " et "élite » s'est transformée en guerre civile larvée dont le dernier épisode, le plus inquiétant, sonne le glas de la dissolution de l'État démocratique. Avec l'autoproclamation de Juan Guaidó comme président par intérim du pays, dans un contexte de crise socio-économique sans précédents dans le pays, on assiste de facto à une déconnexion des pouvoirs démocratiques - exécutif, législatif, judiciaire et militaire -, et à leur mise en tension réciproque. La révolution bolivarienne, qui avait eu une portée radicalement démocratique pour les classes populaires tout au long de la première décennie du $\mathrm{XXI}^{\mathrm{e}}$ siècle, est désormais morte institutionnellement.

Institutionnellement, j'insiste. Le populisme au pouvoir croule sous l'effet de ses nombreuses contradictions, économiques et politiques. Mais rien ne nous assure que ses effets radicalement démocratiques sur les classes populaires disparaissent avec lui. Lorsque j'ai mené mon enquête ethnographique, entre 2007 et 2011, je m'attendais déjà à trouver dans le bastion électoral du chavisme ces mêmes contradictions qui guettaient le populisme au pouvoir : la polarisation, la quête permanente de l'ennemi, 
la banalisation de la violence politique, la réduction de certaines libertés démocratiques. Ce que j'ai trouvé démentait largement mes hypothèses : des habitants des quartiers populaires (barrios) faisant, souvent pour la première fois de leur vie, l'apprentissage d'une démocratie radicale, d'une démocratie fondée sur l'égalité des capacités, des compétences et des droits. Des individus qui luttaient pour se faire reconnaître comme citoyens actifs, qui lisaient et apprenaient par cœur la constitution, qui débattaient passionnément dans leurs assemblées de quartier, qui se sentaient enfin dignes, visibles et écoutés. Les destinataires de l'appel populiste étaient restés, au fond, bien plus fidèles au projet démocratique des débuts que les institutions qui parlaient en leur nom. Le populisme "par le haut» divergeait du populisme "par le bas». Les évolutions institutionnelles du populisme au pouvoir étaient (au moins partiellement) déconnectées de celles, politiques, du populisme dans les quartiers populaires, chez les sujets auxquels le populisme gouvernemental s'adressait.

Mon étonnement avait été fort. Il n'a pas cessé de me travailler par la suite, et ce n'est qu'aujourd'hui, lorsque j'en fais la base d'une nouvelle théorie du populisme, que j'y réponds pleinement. Ce n'est pas cette théorie que je présenterai ici : je me limiterai à reprendre le matériau empirique de cette époque - les observations et les entretiens -, qui avait suscité mon doute méthodique ${ }^{2}$. Ce matériau montre clairement que, en analysant le populisme comme le projet politique d'un État ou d'un parti au pouvoir, les sciences sociales latino-américaines n'en ont vu qu'une face. Cette face est bien connue aujourd'hui : le populisme a été en Amérique latine le moteur d'une réorganisation structurelle de la société «par le haut », par l'État ou le parti au pouvoir, dans le sens d'une démocratisation sociale (di Tella T., 1965 ; Germani G., 1978 ; Touraine A., 1988). La sociologie a appelé cette réorganisation structurelle "intervention nationalepopulaire ». Mais ce n'est là qu'une face de la médaille. Le populisme a été, et continue d'être aussi un phénomène micro-politique, qui affecte le vécu des bénéficiaires du discours étatique et des politiques publiques: ces subalternes qui constituent le «peuple» du populisme au pouvoir. Le populisme n'a pas seulement un « haut », il a aussi un «bas ». À l'instar de l'histoire « from below» d'Edward P. Thompson, il ne faut pas entendre ce «bas » comme l'ensemble des effets sociaux du populisme en milieu populaire: autrement dit, le populisme tel qu'il agit «en bas». C'est surtout le populisme "vu d'en bas » : autrement dit la traduction de la politique populiste dans les catégories de l'expérience populaire.

\section{Les Conseils communaux vénézuéliens comme institutions du "populisme par le bas "}

9 Si l'institution par excellence du péronisme "par le bas » fut le syndicat ouvrier, association de travailleurs, habitants et sujets politiques à la fois (Acha 0., 2004; Martuccelli D. et Svampa M., 1997), celle du populisme vénézuélien a été sans doute le Conseil communal (CC). Créé par le gouvernement bolivarien entre 2002 et 2006, puis réformé en 2009, ce comité local de planification publique s'est moulé sur les anciennes " organisations communautaires de base »: des organisations de quartier qui avaient lutté dans les années 1980 pour des besoins sociaux fondamentaux, comme l'eau, l'assainissement ou l'électricité (Tarragoni F., 2012). Le CC se situait ainsi à mi-chemin entre organisation étatique et organisation populaire : c'était ce statut hybride qui en faisait le réceptacle idéal du populisme au pouvoir, mais également le lieu de traduction 
populaire de ce populisme (Tarragoni F., 2015a). Cela avait été le cas des syndicats péronistes aussi.

Dans les entretiens réalisés entre 2007 et 2011, les CC étaient au cœur d'un renouvellement majeur de la vie politique des quartiers populaires. Leurs habitants s'y engageaient essentiellement pour promouvoir des projets locaux et les faire financer par l'État ${ }^{3}$. Mais ils y voyaient aussi, et surtout, les espaces d'une autonomie collective à construire: il incombait aux CC de faire surgir ce "peuple démocratique » qui, désormais conscient de sa mission historique, devait transformer le pays en binôme avec Hugo Chávez. Toutes proportions gardées, les CC faisaient penser aux comités sans-culottistes de la Révolution française, eux aussi enracinés dans les quartiers populaires et étroitement dépendants d'institutions électives, la Convention et l'Assemblée nationale. Dans cet appel à constituer «un peuple» parmi les classes populaires mobilisées, résidait la véritable clé de voûte du populisme au pouvoir. Et l'appel avait été très performatif: les habitants engagés dans les CC en étaient tous venus à réfléchir à leur potentiel d'émancipation démocratique (Tarragoni F., 2013b). Le populisme les avait amenés à imaginer trois stratégies, indissociablement individuelles et collectives, pour dépasser les rapports de domination dont ils pâtissaient au quotidien.

11 Tout d'abord, ils se sentaient dominés vis-à-vis d'une société qui les rejetait en tant que périphérie jetable de la ville, de la société et de la nation. Aussi ils revendiquaient leur inclusion dans une nouvelle communauté, intégrée et solidaire, dont le peuple était la métaphore. La deuxième expérience de domination qui apparaissait fortement dans les entretiens touchait la question de l'intérêt ou, mieux dit, de l'égoïsme : chacun pour soi et contre les autres. Dans les barrios sévissait la loi de la jungle ; un effet puissant de la précarisation néolibérale des années 1980 et 1990, dont l'émeute du Caracazo (1989) avait été le moment culminant. Le peuple intervenait ici comme figure d'un bien commun à récréer, sur lequel régler des intérêts individuels potentiellement anarchiques et égoïstes. La troisième expérience de domination, enfin, avait trait à l'omniprésence du fatalisme. Les habitants des quartiers populaires étaient, la plupart du temps, résignés à leur destinée sociale, car les chances d'une mobilité, ou même d'un écart par rapport à leur trajectoire prévisible (qu'ils prévoyaient très bien), étaient minimes. Cependant, en tant qu'individus des sociétés modernes (Martuccelli D., 2017), ils étaient profondément travaillés par l'aspiration au bonheur et à l'épanouissement. Avec son appel à l'empowerment, le populisme les incitait à réclamer le droit à se penser comme êtres libres, égaux et singuliers. Le peuple devenait ici une utopie émancipatrice et le populisme une politique de subjectivation.

Le populisme "par le bas" est la somme de ces trois opérations: intégrer la communauté populaire et la faire reconnaître; régler les intérêts individuels sur un commun partageable; se subjectiviser, en tant qu'individus et en tant que collectif.

\section{Le peuple comme métaphore de la communauté}

Dans mon enquête sur la « ville dortoir » de Santa Teresa del Tuy (État Miranda), il était particulièrement évident que le CC avait été investi par les habitants d'une lourde tâche : davantage que faire affluer de l'argent public dans les quartiers, il devait servir à réintégrer une communauté de voisinage menacée d'éclatement. Les habitants s'y engageaient pour reconstituer une vie collective mise en péril par la violence des 
narcotrafiquants et l'arrivée anarchique des réfugiés climatiques de la Tragedia de Vargas ${ }^{4}$. C'est sur ce point qu'insistait en 2007, dès le début de l'entretien, l'un des porte-paroles du CC :

Ce qui se passe ici depuis quelques années, c'est que les gens ont peur de sortir de chez eux. Ils n'ont plus la force de supporter ce qui leur arrive. Nous nous levons tous les jours à $4 \mathrm{~h}$ du matin pour aller travailler à Caracas, nous ne voyons pas nos familles, ni nos enfants. Avant tout ça était encore supportable, parce que nous avions les week-ends et je me souviens qu'il y a encore 10 ans on organisait tout un tas de choses pour la communauté, des rencontres, du cinéma en plein air. On se connaissait, les mères parlaient entre elles des gamins, ils allaient à l'école ensemble. Après la Tragedia, c'est devenu le chaos ici. Les damnificados [victimes de la catastrophe] se sont installés et ont amené la délinquance, la violence. Leurs familles étaient décomposées, ils n'avaient plus rien à perdre. Et ils ont tout détruit. Alors maintenant nous avons peur de nous parler entre nous, nous avons peur de faire jouer les enfants dans les rues. Tu as vu qu'ici après $19 \mathrm{~h}$ il n'y a plus personne dans les rues [Antonio, Entretien, Santa Teresa del Tuy (Miranda), 20075].

Face à ces nouvelles menaces, le leader de la section " animation culturelle et sportive » $\mathrm{du} \mathrm{CC}$, qui vit dans une maison construite avec des matériaux de récupération (un rancho), insiste en revanche sur un détail fondamental :

Contrairement aux autres "comités de sport» du pays, moi je fais jouer tout le monde. Je ne vais pas exclure untel parce qu'il est bizarre, ou parce qu'on l'a vu avec des gens peu recommandables. Je vais organiser des matches de football pour tout le monde. Il y a des gens qui souhaitent jouer avec les autres, il y a des bons, des méchants, des gens qui peuvent être récupérés et avec qui nous pouvons vivre, et d'autres qui ne peuvent pas être récupérés. Mais, même s'il y a des gens méchants dans le barrio, je parle avec eux et je les fais jouer le dimanche; c'est un peu comme ça le CC non? C'est la grande nouveauté de la révolution pour nous, pour moi, de pouvoir parler avec des gens avec qui il serait impossible de parler. Et de faire parler les gens entre eux, tu vois ? [Azoulay, Entretien, Santa Teresa del Tuy (Miranda), 2007].

Azoulay interprète son travail d'animateur sportif du CC comme une mission d'intégration de la communauté. À travers le football, il s'agit de faire rencontrer deux univers séparés, celui des "gens bizarres ou peu recommandables", recouvrant les narcotrafiquants, les familles des victimes de la Tragedia et les voisins stigmatisés, et celui des "habitants méfiants» de la communauté. L'observation des autres CC a largement confirmé cette hypothèse générale : l'une des dynamiques essentielles du populisme «par le bas » n'est rien d'autre que la reconstitution, par le biais de la participation populaire, d'une communauté de voisinage intégrée et solidaire.

Cette poussée intégratrice, que l'on peut penser comme une "communautisation » (Vergemeinschaftung) au sens de Max Weber, comme un processus de production de liens communautaires (Weber M., 2019), ne vise pas que les inassimilables ou les déviants. Son effet est beaucoup plus large et structurant. Dans la majorité des cas analysés, le populisme agit sur une frontière prégnante de l'espace populaire, celle entre les «barrios de fondation » et les "barrios d'invasion ». Les barrios de fondation, plus proches symboliquement de la ville (la ciudad), se caractérisent par un acte de fondation collective et une forte ancienneté des relations de voisinage. Les barrios d'invasion, quant à eux, n'ont pas d'identité propre, ni urbaine ni collective, et sont le produit d'une occupation anarchique de l'espace. Ils sont aux barrios de fondation ce qu'est le barrio à la ville (Bolivar T. et Baldó J., 1995). Habités par les fractions les plus précaires des classes populaires, ainsi que par les migrants et les « aventuriers » de tout 
acabit, ils cumulent, aux dires des habitants des barrios de fondation, toutes les figures de l'anomie sociale'.

17 Mery et Lourdes, deux leaders de deux CC de la banlieue de Caracas (« 23 de enero » et «Petare »), consacrent ainsi une grande partie de leurs entretiens à m'expliquer que les comités permettront aux habitants des barrios d'invasion " qui n'ont jamais participé ", de venir assister aux réunions de leurs quartiers et d'échanger avec leurs voisins :

La communauté « 23 de enero» a été créée dans les années 1950-1960 quand le dictateur Pérez Jiménez a créé ces HLM que tu vois à côté de La Cañada, la grande rue qui longe tout le quartier. À la fin des années 1960 commencent alors à se créer des barrios de fondation, comme le barrio "Santa Rosa » : de 1969 date le premier barrio de fondation à côté de la Avenida Sucre. Des gens venant de la campagne ou chassés de la ville par la spéculation urbaine viennent s'installer dans les barrios avec un projet commun : créer un espace agréable, où les voisins échangent entre eux, avec des places, des lieux communs, sans violence ; un espace pour être entre soi, entre gens connus. [...] À la fin des années 1970, commencent à naître d'autres espaces d'immigration, les barrios d'invasion, comme les barrios "Cristo Rey ", "Camboya ", «Callejón Negro » ou "La Sierra ». Ces barrios sont très différents des nôtres : ce sont plutôt des gens très pauvres, qui n'ont rien à perdre, qui viennent sans famille et qui vont organiser ces espaces de manière sauvage. Ces barrios-là sont très fragiles: tu as vu ce qui s'est passé en décembre dernier, quand une grande partie des barrios de Caracas a été détruite [En décembre 2010, à cause des inondations dans la capitale, de nombreux déplacements de terrains ont détruit des barrios entiers dans l'ouest de la ville]. Ce n'était que des barrios d'invasion. Avant que ces gens-là soient relocalisés dans les différents espaces d'accueil, nous les avions invités à échanger avec nous. Ils étaient venus à la remise des prix du théâtre de rue pour les gamins. On avait discuté des $\mathrm{CC}$, des projets, et on s'était fait une idée un peu les uns des autres. C'était une occasion pour nous de les faire participer à la vie de notre quartier, et inversement tu vois : faire connaître aux voisins ce qui se passe de l'autre côté du quartier, chez eux, leurs problèmes et tout ça. Parce que ces gens-là, tu ne les vois vraiment jamais [Mery, Entretien, 23 de enero (Caracas), 2011].

Les invasions ont commencé ici à la fin des années 1960: on voyait ces gens construire sans aucune règle des maisons, à Petare Norte. [...] Alors quand je commence à m'intéresser au féminisme, au début des années 1970, je vais travailler à Petare Norte. Alors là, c'était un autre monde: des madres solas [mères célibataires] avec je ne sais pas combien d'enfants, qui enfantaient comme des animaux. Moi-même j'étais madre sola, mais des cas de mères avec 5 enfants, ça dans mon barrio on n'en voyait pas. Maintenant, avec les CC, c'est un peu la même chose : il faut se rapprocher de ces situations d'indigence, de violence, d'irrespect des droits fondamentaux. Il faut que les gens de Petare norte viennent travailler avec nous, et que nous allions là-bas les inciter à former des CC [Lourdes, Entretien, Petare (Caracas), 2011].

18 Comme le suggèrent Mery et Lourdes, la frontière spatiale entre barrios de fondation et barrios d'invasion se double d'une frontière symbolique et morale : si les habitants des barrios d'invasion n'ont « jamais participé », c'est que, en réunissent migrants, pauvres, exclus et déviants de toutes sortes, ils vivent dans un état de totale anomie. L'exclusion sociale se double ici d'une représentation morale, qui identifie le « Nous » en excluant, par altérisation, le «Eux» (Elias N. et Scotson J., 1997). Le principal effet des CC a été d'inciter les habitants au dialogue: autrement dit, à récuser toutes ces «logiques d'exclusion » qui conduisent des groupes populaires à marginaliser d'autres groupes populaires, dans des espaces sociaux et spatiaux proches. Il faut intégrer les exclus, les outsiders, les invisibles, pour reconstituer une grande communauté populaire : c'est cette communauté de voisinage élargie que figure, par synecdoque, le peuple du 
populisme. Comme l'écrit Ernesto Laclau, «la construction du "peuple" va être la tentative de donner un nom à la plénitude absente [de la communauté] » (Laclau E., 2008, 106). Dans des quartiers marqués par l'isolement, l'incommunicabilité, la violence et la division, cette « plénitude absente » est un souci crucial des habitants.

\section{Intégrer et faire reconnaître}

Cependant la communauté populaire que le populisme pousse à recréer dépasse largement, dans la plupart des cas, le seul groupe de voisinage. Elle recouvre une forte dimension symbolique : c'est plutôt un idéal de communauté qui permet aux habitants de pointer les nombreuses dynamiques de stigmatisation dont ils souffrent quotidiennement. Cela apparaît clairement dans le discours de Carmen, Robert et José, trois leaders communaux, deux dans la banlieue de Caracas, le troisième dans la périphérie de Valencia :

Ici à Caracas, jamais personne ne s'est intéressé à nous. Le barrio «Santa Rosa ", mais tous les barrios en général, tout le monde, en ville, en a peur. Les taxis n'arrivent pas jusqu'ici, tu le sais. Les gens de l'Est continuent à penser que nous sommes des sauvages. Mais tu sais quoi ? Je pense que c'est eux les sauvages. Nous leur avons montré que nous aussi, nous pouvons avoir des beaux quartiers [barrios bonitos], que nous pouvons aussi nous organiser. Ce sont les CC qui ont fait ça : désormais nous nous sentons meilleurs, nous sentons que nous pouvons nous améliorer, que leur mépris, et tout ce que l'on ressent quand on va en ville, est injuste et injustifié [Carmen, Journal de terrain, 23 de enero (Caracas), 2011].

Je travaille dans un endroit à Caracas où les gens regardent les pauvres comme s'ils étaient des animaux. Dans ce quartier résidentiel, il y a plein de gens qui me parlent des barrios sans savoir que j'y habite aussi, que je viens d'Antimano et que je me suis installé à El Valle. Ils me disent «tous ces marginales [pauvres votant Chávez] qui volent et tout, ils sont tous des malandros [délinquants]». Les gens me racontent un tas de choses horribles, c'est vraiment incroyable. Et moi je ne peux même pas trop dire ce que je pense, je dois tenir ma langue parce qu'il s'agit de mes clients. Ces gens sont normaux j'ai envie de te dire, ils sont aimables, et je crois que s'ils venaient faire un tour dans les barrios voir comment nous nous organisons ici, avec les réunions, les discussions des budgets et tout cela, je crois que le racisme partirait comme un mauvais souvenir. Ce serait pour eux comme un antibiotique, tu vois? [Robert, Journal de terrain, El Valle (Caracas), 2011].

Tu vois ces ranchos là-bas? C'est la partie la plus pauvre du barrio «La Manguita ». Et bien, juste à côté il y a le quartier résidentiel dans lequel tu vis et, encore à côté, le plus luxueux du luxueux, "La Lagunita ». Tu vois ? On vit côte à côte, mais c'est comme si entre nous il y avait un mur infranchissable; d'ailleurs le seul espace de rencontre est la perrera [point de vente d'hot-dog], que je définirais comme une "zone de tolérance sociale»: toutes les classes sociales se réunissent devant un hot-dog, non? Nous connaissons ces gens-là, nous travaillons avec eux, mais nous ne nous sentons pas à l'aise chez eux, et eux ne viennent jamais chez nous. Ils ont peur de nous : dès qu'ils nous voient, ils marchent plus vite, ils se sentent agressés. Et ils ont en partie raison, parce qu'ici dans le barrio, les gens tranquilles vivent mal. Mais maintenant que la communauté a commencé à s'organiser, nous avons la chance de leur montrer que nous ne sommes pas tous des malandros, que nous travaillons pour nous améliorer. Et alors eux ils devraient devenir moins racistes, non? [José, Journal de terrain, La Manguita (Valencia), 2011].

Le populisme "par le bas» ne pousse pas seulement à intégrer la communauté de voisinage, mais aussi à l'exposer à ses dehors et à la faire reconnaître comme une partie 
visible de la société. Le peuple devient ainsi une figure qui soude et dés-altérise, intégre et visibilise. Cette dualité est sa principale force en tant qu'imaginaire politique.

\section{Le peuple comme figure de l'intérêt commun}

21 Un autre effet majeur du populisme " par le bas » est de structurer ce qui permet, audelà des liens de sociabilité, à cette communauté populaire d'exister et de fonctionner. Autrement dit: la conscience d'un intérêt commun, auquel les membres de la communauté tiendraient plus qu'à leurs intérêts particuliers. La première figure du peuple est la communauté ; la deuxième est le commun.

Or, dans les barrios les lieux qui « font commun » sont tragiquement peu nombreux. Il n'y en a souvent qu'un : la place du quartier ${ }^{7}$. Mais il s'apparente davantage à un lieu de passage qu'à un véritable espace de socialisation. Cette absence de commun produit un paradoxe saisissant, qui est au cœur de l'expérience populaire : d'un côté, l'isolement et la communauté de voisinage créent de fortes relations de sociabilité ; de l'autre, les habitants sont toujours renvoyés à leur petit jardin, à leur intérêt personnel, à leur égoïsme, pour la simple raison qu'ils doivent inventer en permanence des stratégies pour survivre à la précarité (comme on dit au Venezuela : " echarle bolas a la vida »). Les quartiers populaires sont tiraillés entre une communauté subjective de vie et une absence objective de commun.

Cette situation interroge profondément les leaders du CC. Ils parlent tous, à un moment ou à un autre, de l'« intérêt personnel » qui régit la vie des quartiers et les éloigne de la politique (ou dénature celle-ci de manière clientéliste). Les $\mathrm{CC}$ les ont poussés à prendre conscience de ce phénomène, à lui trouver des causes et à chercher à le résoudre collectivement. La critique de l'égoïsme sert ainsi, pour chaque habitant engagé dans le $\mathrm{CC}$, à éduquer ses voisins à la condition de peuple. Cette critique n'est pas abstraite : elle est un rouage essentiel du fonctionnement du comité ; celui-ci doit canaliser, en effet, de l'argent public vers des projets d'intérêt commun pour le quartier. Or, pour décider de l'attribution de l'argent, le comité doit suivre une règle de répartition et se prémunir contre toute possibilité de corruption, de favoritisme ou de cooptation. Symétriquement, lorsqu'on demande quelque chose au comité - une allocation alimentaire ou une réhabilitation de la maison -, on doit le faire dans le langage de l'intérêt commun. Ce n'est pas qu'une simple question de rhétorique; c'est une véritable éducation à l'intérêt commun, à cette compétence hautement politique consistant à différencier intérêt individuel et intérêt public.

Ce qu'il faut éviter à tout prix, c'est de passer pour un «individu intéressé » aux yeux des voisins. Et à force de jouer ce rôle, on finit par haïr ceux qui le font. L'assemblée du dimanche est le haut lieu de cette construction politique du commun. Pendant des heures, s'y alternent les demandes et les besoins des uns et des autres; on fait valoir des intérêts personnels mais en portant attention aux demandes d'autrui ; on insiste sur les problèmes de «sa » famille, mais aussi sur ceux d'un voisin « connu par tous » et vivant dans le dénuement; on accepte de mettre provisoirement de côté son besoin immédiat pour satisfaire celui, plus pressant, que la communauté a jugé prioritaire (Tarragoni F. et Vilas Boas M.-H., 2015b). La première impression que j'ai eue en assistant à une assemblée du $\mathrm{CC}$, a été de vivre une gigantesque mise en scène du Contrat social de Rousseau. Tout se passait comme si le concept philosophique de volonté générale prenait corps devant moi. 


\section{L'apprentissage du droit} besoins individuels ou familiaux, à la situation d'autrui. En entendant les demandes des uns et des autres, chaque individu est familiarisé avec la «carte des besoins» du quartier, et peut s'y positionner rationnellement. En amont de chaque projet à financer, les débats doivent permettre à cette "carte des besoins " d'être visible par tous. Chaque voisin doit être mis en condition de comparer ses propres besoins à ceux des autres, à la moyenne des besoins insatisfaits de la communauté, et donc à ce qu'on peut faire en priorité. C'est la conversion de l'individu égoïste en voisin à l'écoute, et de celui-ci en acteur débattant à l'assemblée, qui résume le processus de construction du «peuple » par le bas.

Le droit revêt un rôle central dans ce processus. Il permet en effet de partager ontologiquement le privé (donc l'appropriable) et le public (le non-appropriable). On en trouve deux exemples très éclairants dans l'« affaire du camion pour les déchets ", qui déchire le CC paysan de Misintá, et dans les discussions entre élus et experts sur le «statut juridique » de l'aqueduc en construction à Bello Monte (Mérida) :

Quand on a essayé de nous voler ce camion que le CC avait financé pour régler l'histoire des déchets, ça a été malgré tout un moment très important pour la communauté. Un moment central de la vie de la communauté. Tu me diras: comment un vol peut-il être un moment positif de la vie communautaire ? Ce n'était pas tellement le vol en soi, qu'on a réglé après. On a découvert les coupables, qui ont fait amende honorable devant la communauté. L'important a été le débat qui a suivi. Parce que toute la communauté a pu discuter autour de cette question : «à qui était vraiment ce camion? ». Alors nous, élus du CC, nous avons lancé le débat, parce qu'il y avait un type, le fils d'un paysan qui vit en bas de la route, vers la ville, qui a dit que du moment où on avait besoin d'un camion et que le besoin le justifiait, on pouvait l'emprunter à la communauté. Alors je lui ai dit « Tu penses vraiment qu'on peut emprunter à la communauté? Tu penses qu'on peut s'approprier les biens d'une communauté comme ça impunément?». Parce que c'est de cela qu'il s'agit. Les gens qui ont fait ça se sont appropriés un truc qui était à tout le monde, et maintenant ils doivent assumer la responsabilité d'avoir fait un truc contre la loi de la communauté. Et les autres membres de la communauté ont réagi, c'était super, chacun avec sa vision des choses. Un vrai débat sur comment on décide de s'organiser ici, sur ce qu'on fait des besoins des gens, sur ce qui appartient à tous, à moi, à toi, à tout le monde, et à personne en particulier. [Giorman, Entretien, Misintà (Mérida), 2011].

Il est très important de donner un statut juridique à cet aqueduc. Parce que comme on va demander aux gens de la communauté de le construire selon la modalité du travail socialiste [travail gratuit et coopératif], après, une fois qu'il sera construit, il faudra que tout ce travail soit bien valorisé. On ne pourra pas permettre que des gens fassent des dégâts ou se l'approprient de manière privative. C'est fondamental. Alors je ne sais pas, il faut trouver une manière légale, il faut mettre ça dans un règlement du $\mathrm{CC}$, pour que les gens comprennent que ce n'est pas à eux, mais que ça appartient à tout le monde. D'ailleurs les voisins de la partie sud du barrio disent que, selon eux, il faut écrire tout cela très clairement dans un code de conduite supplémentaire qui ait valeur d'engagement pour tous, et qu'on mettrait dans le projet pour le MINCOM (Ministère des Communes) [Intervention du « Professeur » à l'Assemblée, Journal de terrain, Bello Monte (Mérida), 2011].

Comme en témoignent ces extraits, les moments du vol du camion et de la construction de l'aqueduc ont été des événements structurants du «peuple » de la communauté : les 
habitants ont participé activement au débat sur l'appropriable et le non-appropriable, sur le privé et le commun. Or, ces discussions sont monnaie courante des assemblées. C'est pourquoi les habitants, pour délibérer en commun, se forment de plus en plus aux logiques du droit. S'ils lisent la constitution pendant leur sieste poméridienne ou avant de se coucher, si on trouve partout dans les barrios des vendeurs ambulants de textes juridiques et décrets-lois, ce n'est pas parce que le peuple aime viscéralement l'État bolivarien et tout ce qu'il produit de lois. C'est parce que le droit revêt une fonction fondamentale dans la politisation populaire : il permet de faire valoir un besoin, de le construire en besoin légitime, de distinguer ce qui « appartient à tous, à moi, à toi, à tout le monde, et à personne en particulier ». Bref, il permet de construire un peuple par la participation.

\section{Le peuple comme opérateur de subjectivation} «subjectivisant" de leur engagement. Le sociologue avide de matérialité, de " concret ", et soucieux de briser les illusions des «agents", pourrait être tenté de passer un voile sur ces dynamiques de subjectivation, n'y voyant que le vernis rhétorique ou justificatif que les individus apposent sur leurs stratégies intéressées dans le comité. Et pourtant cette opération s'avère un pis-aller interprétatif : comment expliquer en effet que tous les interviewés décrivent de manière extraordinairement précise les émotions ou les épiphanies personnelles vécues suite à leur engagement dans le comité ? Répondre à cette question nous conduit à apporter une ultime touche au «peuple " généré par le populisme chaviste : un peuple qui, en plus d'être le nom d'une communauté intégrée et solidaire et de l'intérêt commun sur lequel cette communauté est bâtie, est aussi l'opérateur d'une émancipation individuelle et collective.

La subjectivation trouve son origine dans cet appel à l'empowerment que le populisme lance en milieu populaire. Lorsque le leader charismatique enjoint les subalternes à «se faire peuple », l'appel est non seulement pris au sérieux, mais devient très performatif. Les CC deviennent des institutions permettant de le réaliser. En participant, en prenant la parole, en s'indignant, l'habitant se transforme. Ces pratiques politiques s'imbriquent chez lui ou chez elle à des ruptures biographiques préalables, en les amplifiant (Tarragoni F., 2015c, 150-165). Que les épreuves que l'habitant a à surmonter dans sa vie personnelle - et elles sont nombreuses - soient familiales ou professionnelles, scolaires ou migratoires, liées à sa santé ou à celle des proches, c'est l'engagement dans le comité qui le dote de nouveaux supports, de liens, de raisons de continuer à vivre. Prenons un cas assez fréquent pour les leaders des $\mathrm{CC}$, qui sont souvent des femmes : celui d'une maternité non choisie, à gérer dans la solitude. C'est le cas de Lorena, élue d'un quartier indigène de la Guajira (wayúu) qui me raconte avoir décidé de s'engager dans le $\mathrm{CC}$ après avoir croisé ses voisines aux ateliers de planning familial :

Moi, j'ai trois enfants [l'enquêtée a 19 ans]. J'ai fait le premier à 14, le deuxième à 15 et le troisième à 17 . Beaucoup eh [en réagissant à mon étonnement] ! Je sais... Mais pour le moment c'est fini. Je m'occupe de ma famille et c'est tout. Heureusement qu'il y a ma mère et Euclide pour m'aider. Là j'ai commencé à participer aux séances de l'atelier de planning familial, et je me suis rendue compte que j'étais un peu bête de faire ça. Parce que tu te fais prendre par l'inconscience et tu fais un enfant. Nous 
femmes, ça nous arrive souvent ça. Alors que ce n'est pas bien, ni pour moi, ni pour l'enfant. Mais, tu vois, c'est là que je me suis dit que la participation c'était bien, parce qu'avant je n'avais jamais réfléchi à ça : mes voisines m'ont ouvert les yeux. Et discuter avec elles dans le planning familial, ça m'a permis d'aborder des choses dont je ne parle jamais dans la vie de tous les jours. Tu prends ce qui t'arrive et tu ne réfléchis jamais vraiment. Maintenant je fais comme les autres, nous sommes là à discuter, nous nous sentons plus fortes, nous pouvons faire des choses qu'avant on n'aurait pas imaginé de pouvoir faire [Lorena, Journal de terrain, Guajira (région indigène), 2011].

L'entrée dans l'atelier de planning familial a eu l'effet d'une révolution subjective : avec ses voisines, Lorena a pu discuter de « choses dont elle n'avait jamais parlé dans la vie de tous les jours » et s'est rendue compte qu'elle pouvait « faire des choses qu'avant on n'aurait pas imaginé de pouvoir faire ». Il est très symptomatique qu'elle reconnaisse, d'entrée de jeu, que mettre des problèmes en commun et les exposer au regard public, est une manière de "réfléchir à ce qui t’arrive " et de se découvrir des capacités insoupçonnées (Tarragoni F., 2014). Souvent ces femmes qui s'impliquent dans les CC décrivent leur engagement comme une mise au service de la communauté de compétences vouées, dans le cadre domestique, à l'invisibilité et à l'illégitimité. Il s'agit, pour reprendre le vœu de nombre d'entre elles, de se mettre au service du peuple ; et ce faisant, de retirer de la participation une image valorisante d'elles-mêmes.

Mais afin d'expérimenter de nouvelles capacités, l'habitant doit avant tout reconnaître de manière réflexive, puis mettre à distance, l'ensemble des dominations (culturelle, éducative, politique) qui pèsent sur son existence. Revenir sur la passivité, le fatalisme, l'incapacité de naguère est la seule manière de prendre conscience de la nouveauté radicale de ce que l'on vit avec le populisme. C'est ce que souligne Freddy :

Quand je suis allé pour la première fois à l'Assemblée, je ne comprenais pas grande chose. Je n'ai jamais rien compris à la politique de toute façon. Ça n'a jamais été un truc important pour moi, tu vois? Mais bon, là je me suis dit : il y a plein de voisins qui y vont, plein de gens qui se présentent; voyons un peu à quoi ça rime. Et au début, on parlait de choses très techniques, de matériaux à acheter, de fiches de réhabilitation des maisons à remplir, d'échéances fixées par la mairie, de budgets. Je ne savais pas de quoi on parlait, je te l'avoue. J'étais un peu largué. Maintenant je n'ai pas l'impression d'en savoir techniquement beaucoup plus, mais j'ai compris pourquoi nous parlons de tout ça. Nous parlons de ça parce que nous pouvons le faire ensemble, chacun avec ses compétences, chacun avec ses qualités. Alors Branger nous dit comment faire un projet et nous l'écoutons ; après nous lui disons comment écrire le projet, ce qu'il va mettre dedans, parce que seulement nous, en tant qu'habitants, savons ce dont nous avons besoin. Nous apprenons un peu de technique : là, tu vois, je suis en train d'apprendre la compatibilité et toutes ces choses, et les autres apprennent de nous. C'est ça qui te fait sentir que tu comptes, que t'es quelqu'un, que t'es une personne comme les autres, après une vie passée à vivre comme des animaux ici dans les barrios! [Freddy, Journal de terrain, 23 de enero (Caracas), 2011].

Freddy commence son récit par l'expérience d'une incapacité et d'une incompétence. Il avoue avoir été " un peu largué » dans un premier temps. Ce n'est qu'en revenant sur les raisons de cette incapacité, et en se donnant les moyens d'apprendre ce qui lui faisait défaut, qu'il a pu, d'une certaine manière, la dépasser. L'expérience de ce dépassement produit alors une nouvelle subjectivité. Freddy sent désormais qu'il " compte quelque chose, qu'il est quelqu'un, qu'il est une personne comme les autres, après une vie passée à vivre comme des animaux dans les barrios ». Cette subjectivité nouvelle est faite, comme on le voit aisément, de latences de l'émancipation dans une 
trajectoire singulière : Freddy s'est désormais découvert «autre » que ce qu'il pensait être, c'est-à-dire autre que ce qu'il était lorsqu'il pensait ne pouvoir être rien d'autre. Le CC dit aux habitants : prenez la parole, éprouvez la liberté et émancipez-vous! Autrement dit: devenez un peuple! C'est en ce sens que le populisme produit de la subjectivation politique parmi les subalternes: il les conduit à se forger un nouveau rapport à soi qui les valorise à mesure qu'ils se sentent part d'un collectif virtuel qui les dépasse - le peuple précisément.

\section{S'émanciper sur fond de précarité} interne, qui apparaissait pleinement dans les entretiens de 2011. Toujours sous l'emprise de la précarité, les barrios faisaient avec le populisme l'expérience d'un nouveau possible. Mais la précarité n'avait pas disparu en un éclair. Bien qu'elle fût lézardée par les effets émancipateurs du populisme, elle persistait. Elle subsistait dans la fragilité économique et l'incertitude professionnelle des vies populaires, ainsi que dans le fatalisme qui en découlait, très présent dans les entretiens. Ce fatalisme entrait en contradiction avec le sentiment d'une puissance retrouvée : tout se passait comme si le populisme accentuât tragiquement, tout à la fois, le potentiel émancipateur de la politique dans les quartiers populaires, et la difficulté de la politique à changer quoi que ce soit ; comme s'il ouvrait un nouveau chemin, tout en pointant à quel point il était difficile à parcourir.

Les leaders du CC évoquaient souvent, en guise de conclusion des entretiens, cette contradiction. Ils en faisaient la principale aporie du nouveau peuple qu'ils étaient chargés de créer en duo avec Chávez. Plus que l'isolement des quartiers ou la persistance de l'égoïsme, le véritable obstacle, disaient-ils, à la construction d'un peuple dans les barrios était la résilience d'une culture de la précarité, du fatalisme, de la passivité, de l'abandon de soi :

Alors Chávez et tous les autres, les gens du Ministère, ils n'arriveront jamais à résoudre ça. Le fait que, quoiqu'on fasse, on n'arrive pas à être un peuple. Je te le dis : les CC ne fonctionneront jamais si nous n'arrivons pas à construire la culture socialiste. Parce qu'ici, la culture d'avant, la culture puntofijista [du nom de la démocratie de Punto Fijo, née en 1961 et enterrée par la révolution bolivarienne ; ce système politique était basé sur l'alternance du parti social-démocrate et du parti social-chrétien, et sur l'exclusion du parti communiste], règne souveraine et les gens ne seront jamais libres avec cette culture. Les gens ont les taudis (ranchos) dans la tête! Alors ils essaient, et l'essai vaut déjà quelque chose, mais après ils retombent dans l'indifférence, dans le fatalisme, dans leur petit foyer. Et ils arrêtent de penser aux autres. Et ce n'est pas seulement un problème d'argent public, ou de transparence du CC. C'est un problème de culture, cette culture dans laquelle nous baignons depuis toujours dans les barrios [José, Journal de terrain, La Manguita (Valencia), 2011].

«Les gens ont les ranchos dans la tête! ». Autant dire que, malgré leur projection vers un idéal émancipateur, les habitants éprouvent toujours la force de rappel de la précarité. Force de rappel d'un mode de vie qui, métaphorisé par les "ranchos", renvoie aux difficultés économiques persistantes, à l'incapacité de s'en sortir et de se projeter, au cumul des échecs, à l'incertitude professionnelle et familiale, à la violence du narcotrafic et de la police. Cette condition de vie, aux antipodes de la «vita activa» dont parlait Hannah Arendt (2002, 17-35), impose, en un certain sens, de penser à soi 
contre les autres. Le peuple apparaît ainsi dans l'écart entre ces deux forces opposées et pourtant indissociables, l'élan vers l'émancipation et la force de rappel de la précarité. Il désigne la communauté politique qui prend forme «sous» le populisme, mais qui reste toujours à faire. Il fait figure d'interstice entre la communauté populaire déjà là, celle qui "a les ranchos dans la tête ", et le collectif politique à faire, composé des voix, des agir et des revendications des uns et des autres.

Ce populisme par le bas semble, à la lumière de l'actualité du pays, tragiquement derrière nous. Mes enquêtes datent d'il y a dix ans ou presque : tout laisse imaginer que l'expérience politique populaire a profondément évolué avec la crise économique, les pénuries, l'hyper-inflation. Mais il ne reste pas moins qu'une telle politisation démocratique, sur la durée, a laissé des traces indélébiles dans les classes populaires. C'est à partir de ces traces qu'il faut essayer de comprendre, de manière sociologiquement rigoureuse, la nature du populisme comme mode d'action politique.

\section{BIBLIOGRAPHIE}

Acha, Omar, « Sociedad civil y sociedad política durante en primer peronismo », Desarrollo Económico, n 174, 2004, p. 199-229.

Arendt, Hannah, Condition de l'homme moderne, Paris, Pocket, 2002 (1ère éd. 1958).

Bolivar, Teolinda et Baldó Josefina (eds.), La cuestión de los barrios, Caracas, Monte Ávila Editores/Fundación Polar UCV, 1995.

Canovan, Margaret (ed.), Populism, New York/London, Harcourt Brace Jovanovich, 1981.

Dézé, Alexandre, « Le populisme ou l'introuvable Cendrillon. Autour de quelques ouvrages récents ", Revue française de science politique, vol. 54, n 1, 2004, p. 179-190.

Elias, Norbert et Scotson, John L., Logiques de l'exclusion. Enquête sociologique au cœur des problèmes d'une communauté, Paris, Fayard, 1997 (1ère éd. 1965).

Germani, Gino, Authoritarianism, Fascism, and National Populism, London/New Brunswick, Transaction Books, 1978.

Gramsci, Antonio, Nel mondo grande e terribile. Antologia degli scritti 1914-1935 (éd. Giuseppe Vacca), Turin, Einaudi, 2007.

Hermet, Guy, Les populismes dans le monde. Une histoire sociologique xixe-xxe siècle, Paris, Fayard, 2001.

Ionescu, Ghita et Gellner, Ernest (eds.), Populism. Its Meaning and National Characteristics, London, Weidenfeld \& Nicholson, 1969.

Laclau, Ernesto, La raison populiste, Paris, Seuil, 2008 (1ère éd. 2005).

Martuccelli, Danilo et Svampa, Maristela, La plaza vacía. Las transformaciones del peronismo, Buenos Aires, Losada, 1997. 
Martuccelli, Danilo, La condition sociale moderne. L'avenir d'une inquiétude, Paris, Gallimard, 2017.

Merklen, Denis, Asentamientos de la Matanza. La terquedad de lo nuestro, Buenos Aires, Catálogos Editora, 1991.

Müller Jan-Werner, Qu'est-ce que le populisme ? Définir enfin la menace, Paris, Gallimard, 2018.

Quattrocchi-Woisson, Diana, « Les populismes latino-américains à l'épreuve des modèles d'interprétation européens », Vingtième siècle, nº 56, 1997, p. 162-163.

Revet, Sandrine, Anthropologie d'une catastrophe. Les coulées de boue de 1999 au Venezuela, Paris, Presses Sorbonne Nouvelle, 1999.

Taguieff, Pierre-André, L'illusion populiste. Essai sur les démagogies de l'âge démocratique, Paris, Champs Flammarion, 2007.

Tarragoni, Federico, « Comprendre des peuples organisés. Les Consejos comunales de planificación pública et la diversité populaire de la politique », Terrains et travaux, $n^{\circ} 21,2012$, p. $145-164$.

Tarragoni, Federico, « La science du populisme au crible de la critique sociologique : retour sur l'archéologie d'un mépris savant du peuple », Actuel Marx, n54, 2013a, p. 56-70.

Tarragoni, Federico, « Raison populiste, démocratie et émancipation populaire », in Hadrien Buclin, Joseph Daher, Christakis Georgiou et al. (dir.), Penser l'émancipation, Paris, La Dispute, 2013b, p. 215-234.

Tarragoni, Federico, « De la personne au sujet politique. Une ethnographie des prises de parole populaires dans les assemblées de barrio au Venezuela », Participations, vol. 9, n², 2014, p. $149-175$.

Tarragoni, Federico, «Entre dépendance et rêve d'autonomie : les usages populaires de l'État au Venezuela contemporain », Politix, vol. 29, no 110, 2015a, p. 171-193.

Tarragoni, Federico et Vilas Boas, Marie-Hélène, « Le concept de clientélisme résiste-t-il à la participation populaire ? Une comparaison Brésil-Venezuela ", Critique internationale, $n^{\circ} 67$, 2015b, p. 103-124.

Tarragoni, Federico, L’Énigme révolutionnaire, Paris, Prairies ordinaires, 2015c.

Tarragoni, Federico, « La méthode d'Edward P. Thompson », Politix, vol. 30, n 118, 2017, p. 183-205.

Tella, Torcuato S. (di), « Populism and Reform in Latin America », in Claudio Veliz (ed.), Obstacles to Change in Latin America, Cambridge, Cambridge University Press, 1965, p. 47-74.

Touraine, Alain, La Parole et le sang. Politique et société en Amérique latine, Paris, Odile Jacob, 1988.

Verret, Michel, L'espace ouvrier, Paris, Armand Colin, 1979.

Villacañas Berlanga, José Luis, Populismo, Milano, Mimesis, 2017 (1ère ed. 2015).

Weber, Max, Les communautés (tr. et éd. C. Colliot-Thélène et É. Kauffmann), Paris, La

Découverte, 2019. 


\section{NOTES}

1. À cet égard, les populismes latino-américains réussirent à instrumentaliser les passions nationales dans le sens du combat anti-impérialiste, combat internationaliste par excellence. Dans les années 1930, Antonio Gramsci préconisait quelque chose de similaire pour l'Italie, empêtrée dans l'ultranationalisme impérialiste mussolinien. Il soulignait que, depuis le Risorgimento du XIXe siècle, en raison de la place périphérique de l'Italie dans la division internationale (capitaliste) du travail, et de l'apparition d'un " colonialisme interne » du Nord vers le Mezzogiorno, «le peuple italien est [...] "nationalement" l'un des plus intéressés à une forme moderne de cosmopolitisme", qu'il identifie avec l'internationalisme prolétarien (Gramsci, 2007, p. 182, ma traduction).

2. Ce matériau empirique a été réuni dans un livre, Le peuple et le caudillo. La question populiste en Amérique latine contemporaine, dont l'édition a été confiée aux Presses Universitaires de Rennes. En raison d'un aléa de financement, cet ouvrage, pourtant finalisé et revu par l'éditeur, n'a jamais vu le jour. Je profite de l'occasion qui m'est donnée ici pour en publier partiellement son volet empirique, la partie plus théorique ayant été inclue dans mon livre L'esprit démocratique du populisme. Une nouvelle analyse sociologique (La Découverte, coll. «L'horizon des possibles »).

3. Les CC ont été introduits dans le cadre d'un changement plus global de l'architecture des politiques publiques dans le pays, en vue de favoriser un processus de décentralisation, de déconcentration de l'État et de responsabilisation des bénéficiaires de l'action publique. Chaque comité possède un périmètre réglementaire de 200 familles (hors territoires indigènes), et se trouve en relation directe avec le Ministère de la participation et du développement social (MINPADES, désormais Ministère des Communes, MINCOM). Celui-ci est, par l'intermédiaire de FUNDACOMUNAL, le principal bailleur des fonds, issus pour l'essentiel de la rente pétrolière. Avec la Loi de 2009, ces comités se dotent d'une nouvelle institution, la Sala de batalla electoral, organisme de "conscientisation idéologique » qui pousse de plus en plus les élus locaux à s'affilier au parti chaviste, le Partido socialista unido de Venezuela (PSUV). Ainsi tous les porteparoles des CC, dont on écoutera par la suite les prises de parole, sont au moment de l'enquête des militants du PSUV, bien que leurs relations avec l'idéologie chaviste puissent aller d'une loyauté inconditionnelle à une adhésion purement " pragmatique ».

4. Catastrophe naturelle s'étant produite en décembre 1999 par des inondations et des coulées de boue sur le littoral caraqueño (Revet, 2007).

5. Nous ne donnons pas, dans le cadre de cet article, d'indications sociographiques supplémentaires sur les enquêtés, car elles ne sont pas utilisées par la suite. Les interviewés font tous partie des "classes populaires", qui ont, au Venezuela et plus largement dans le souscontinent, une consistance sociologique autrement plus forte qu'en Europe ou en France. Elles se définissent à partir de trois critères : la ségrégation spatiale (leur assignation au barrio), le primat du marché du travail informel et la domination symbolique (elles sont assimilées à la barbarie). Cependant, à l'intérieur de cet ensemble plutôt homogène, les porte-paroles des CC tendent à former une micro-élite, en raison de leurs capitaux scolaires et militants. C'est le cas de tous les interviewés.

6. Dans son travail sur les occupations illégales des terrains dans la banlieue de Buenos Aires, Denis Merklen (1991) a pointé un phénomène tout à fait similaire pour le cas argentin : les bâtisseurs des barrios « de fondation » opposaient leur statut de «bons voisins " (buenos vecinos) à la barbarie des villeros (les habitants des bidonvilles).

7. Pour une analyse similaire à propos de la structuration des espaces ouvriers, cf. M. Verret (1979). 


\section{RÉSUMÉS}

Le populisme continue d'être manié dans les médias et une partie des sciences sociales comme un concept flou, synonyme, tour à tour, de nationalisme, démagogie ou xénophobie. Face à cette inconsistance conceptuelle, l'Amérique latine reste un point de passage obligé. Le populisme fait pleinement partie de son histoire politique, et les sciences sociales continentales ont cherché à en donner une définition rigoureuse et comparative. Force est de constater, toutefois, que la sociologie en est restée souvent à une approche en surplomb : elle en a fait un trait idéologique des États, des politiques publiques, des organisations politiques. À l'aide d'une enquête de terrain menée dans les quartiers populaires vénézuéliens entre 2007 et 2011, cet article interroge, au contraire, les modes de politisation sous-jacents au populisme. Il cherche à comprendre comment un populisme se légitime et se reproduit dans la vie sociale à travers l'action de ces mêmes individus que les politiques populistes ciblent: les habitants des quartiers populaires. On montrera que ce populisme " par le bas » correspond à un certain type d'expérience politique : une certaine manière d'intégrer la communauté populaire et ses dehors, de régler les intérêts des habitants par rapport à une vision du bien commun, et de lier, pour chaque individu, un appel inédit à l'émancipation et l'héritage écrasant de la précarité.

Populism continues to be used in the media and part of the social sciences as a vague concept synonymous with nationalism, demagogy or xenophobia. In the face of this conceptual inconsistency Latin America remains a case in point: populism is an integral part of its political history, and the continental social sciences have sought to define it rigorously in a comparative perspective. It must be noted, however, that Latin American sociologists have often considered populism "from above" as an ideological trait of States, public policies and political organizations. Based on a field survey conducted in the Venezuelan working-class communities (barrios) between 2007 and 2011, this article seeks, by contrast, to examine the modes of politicization underlying populism so as to understand how it legitimizes and reproduces itself in social life through the actions of the very same individuals that are targeted by populist policies -the inhabitants of working class neighborhoods. The aim is to demonstrate that populism "from below" corresponds to a certain type of political experience, i.e. a certain way of integrating community both from within and from without; of regulating the interests of the community members in relation to an ideal of the "common good"; and of instilling in each individual an unprecedented call to emancipation while acknowledging the crushing legacy of precariousness.

El populismo sigue siendo analizado en los medios de comunicación y parte de las ciencias sociales como un concepto indeterminado, sinónimo de nacionalismo, demagogia o xenofobia. Frente a esta inconsistencia conceptual, América Latina sigue siendo un contexto sociopolítico fundamental: el populismo es parte de su historia política, y las ciencias sociales han tratado de construir una definición rigurosa y comparativa. Sin embargo, la sociología se ha limitado, en la mayoría de sus análisis, à un enfoque puramente "macro" del fenómeno. A menudo se ha convertido el populismo en un rasgo ideológico de los Estados, de las políticas públicas o las organizaciones políticas. A través de un trabajo de campo realizado en los barrios venezolanos entre 2007 y 2011, este artículo cuestiona los modos de politización que subyacen al populismo. Busca comprender cómo se legitima y se reproduce el populismo en la vida social a través de la acción de estos mismos individuos a los que se dirigen las políticas populistas: los habitantes de los barrios populares. Agregaremos que este populismo "desde abajo" corresponde a un cierto tipo de experiencia política: el populismo aparece para integrar a la comunidad popular y su exterior, regular los intereses de los habitantes en relación con una visión del bien común, y 
vincular, para cada individuo, un nuevo llamado a la emancipación y el aplastante legado de la precariedad.

\section{INDEX}

Mots-clés : populisme, participation, démocratie, barrios, subjectivation politique

Palabras claves : populismo, participación, democracia, barrios, subjetivación política

Keywords : populism, participation, democracy, barrios, political subjectivation

\section{AUTEUR \\ FEDERICO TARRAGONI}

Federico Tarragoni est maître de conférences en sociologie à l'Université Paris 7-Denis Diderot et agrégé de sciences sociales. Chercheur au Laboratoire du changement social et politique (LCSP), il dirige le Centre de recherches interdisciplinaires sur le politique (CRIPOLIS) auprès de l'Institut Humanités, Sciences et Sociétés de Paris Diderot. Ses domaines de recherches incluent l'étude des processus révolutionnaires et des mouvements sociaux à l'échelle individuelle et biographique, ainsi que l'analyse du populisme entre l'Amérique latine et l'Europe. Il a publié L'Énigme révolutionnaire (Prairies Ordinaires) et Sociologies de l'individu (La Découverte), et publiera, en 2019, L'Esprit démocratique du populisme (La Découverte). 